\title{
Intermodulation distortion analysis of a complex signal in a microwave optics transmission line
}

\author{
Andrey D. Shcherbinin ${ }^{1,2}$, Leonid A. Belov ${ }^{1,2, *}$, and Alexander S. Kondrashov ${ }^{2}$ \\ ${ }^{1}$ National Research University «Moscow Power Engineering Institute», 111250, Moscow, Russia \\ ${ }^{2}$ Joint Stock Company Russian Space Systems, 111250, Moscow; Russia
}

\begin{abstract}
The requirements for RF fiber optic links for placement on board an Earth satellite or other spacecraft [1-3] are distinguished by a combination of additional hard indicators: by weight, overall dimensions; energy efficiency of the power supply; radiation resistance; reliability of long-term autonomous functioning; out-of-band emission level; cost. Therefore, the development of such devices is relevant and in demand.
\end{abstract}

\section{Introduction}

The presence of an ultra-wide frequency band leads to the transmission of the components of the fiber optic communication line (FOCL), as shown by the results of measurements of the level of IMR with a two-frequency test input exposure at frequencies $f_{1}=f_{0}-\Delta / 2, f_{2}=f_{0}+\Delta / 2$ in the vicinity of the central odds $f_{0}$ with frequency spacing $\Delta$ odd-order spectral components of intermodulation distortion (IMD) absent in the input signal from the FOCL with frequencies $\left(2 f_{1}-f_{2}\right),\left(2 f_{2}-f_{1}\right),\left(3 f_{1}-2 f_{2}\right),\left(3 f_{2}-2 f_{1}\right), \ldots$ near the center frequency $\mathrm{f0}$ and its harmonics, and components with frequencies $\left(f_{1} \pm f_{2}\right), 2\left(f_{1} \pm f_{2}\right), \ldots$. The relative power level of the latter takes into account the compensation in the modulator of the symmetrical components due to the balance of the phase relations due to the parity of the combination on both sides of the frequency value of the optical center frequency $f_{0}$.

\section{Measurements}

Fig. 1 shows a block diagram of an assessment of the level of intermodulation distortion of radio signals in a fiber line using a two-frequency technique.

The test results showed:

a) for a short FOCL with direct modulation of the light intensity of the red photodiode (f0 $\approx 294 \mathrm{THz}, \lambda \approx 760 \mathrm{~nm}$ ) in the low-signal mode, the depth of the interference-free dynamic measurement range of the SFDR noticeably exceeds $70 \mathrm{~dB}$;

*Corresponding author: belovla@gmail.com 


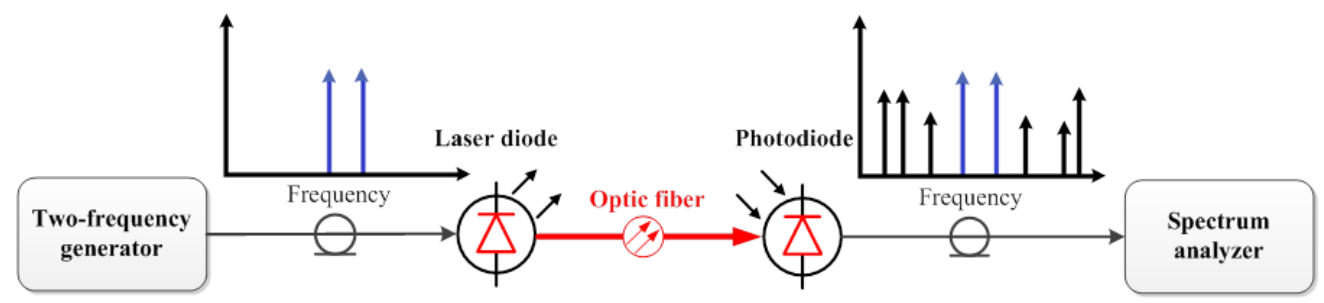

Fig. 1. Structural diagram for assessing the level of intermodulation distortion of radio signals in a fiber line using a two-frequency technique.

b) with an increased power level of the input test two-frequency signal, which leads to gain compression near the saturation (reduction of the transmission coefficient) by $3.5 \mathrm{~dB}$, the relative level of the third-order IMR components monotonically increases;

c) the second-order IMR level at the indicated gain compression value is an unacceptably high level of $-15 \mathrm{~dB}$, and of a higher odd order it pulsates with increasing modulation depth, reaching $-35 \mathrm{~dB}$.

\section{Distortion of microwave signals in on board satellite RF-FOCL}

The development of fiber-optic communication lines for on-board satellite equipment requires taking into account [4-6] the influence of the manifestations of chromatic and polarization dispersion characteristic of the optical range in the band of used optical frequencies, noise, and the use of measures to linearize the nonlinear amplitude, phasefrequency, and intermodulation characteristics of the transmitted microwave signal in the components of the fiber-optic cable ( laser diode, modulator, fiber, photodetector, microwave power amplifier).

The transfer of an information microwave signal to an optical carrier wave and its inverse recovery in an onboard FOCL is accompanied by noise and nonlinear [7] (amplitude, phase, polarization) distortions and noise in the light source, modulator, fiber, and photodetector.

On-board radiofrequency fiber-optic communication lines (RF-FOCL) are designed for:

a) supporting the operation of a reference oscillation source with a precision small frequency instability on board the spacecraft;

b) the formation in the nodes of the on-board equipment of clock signals of the UHF or microwave ranges with a phase error of units of degrees and time stamps for global satellite navigation aids;

c) ensuring the time-frequency synchronization of satellite constellation and groundbased means of a single exact time (MSET) using inter-satellite laser lines;

d) microwave signal routing in airborne broadband relay complexes with a large number of receiving-transmitting trunks;

d) ensuring reliable transmission of telemetry, control and data signals inside the spacecraft and to the ground control complex.

Compared to main FOCLs, on-board satellite fiber lines are distinguished by their use inside the spacecraft:

a) FOCL of relatively short length (up to $30 \mathrm{~m}$ );

b) the limited width of the frequency spectrum of the transmitted information (up to 40 $\mathrm{GHz}$;

c) using a single for all subsystems source of reference frequencies and clock time stamps [4]; 
d) single-channel transmission of analog radio-frequency or two-level telemetric signals and control commands.

A precisely high level of stability of the frequency of the reference source is necessary for communicating with remote spacecraft, for solving the problems of relaying large flows of information, television broadcasting and organizing long-distance communications. When transmitting reference signals for the time-frequency synchronization of satellite constellation and terrestrial services, reference signals of time and frequency of $5 \mathrm{G}$ communication networks, radio navigation equipment and special purposes, it is necessary to ensure the possibility of [8] comparing accurate time scales with an error of not more than $100 \mathrm{~ns}$ at time intervals of up to a day. For this purpose, an atomic or optical frequency standard with an average quadratic relative frequency instability of about 10-17 at an averaging time of up to $104 \mathrm{~s}$ is placed on board the spacecraft. Precise time signals along inter-satellite optical lines synchronize processes on all the spacecraft and are transmitted to ground-based points of the MSET.

Nonlinear effects in FOCL components [4] are determined by the choice of the type and power level of the laser source, as well as the method of inputting the information modulating signal. The reasons for the appearance of an unacceptable level of noise, dispersion, and nonlinear intermodulation distortions of transmitted microwave signals are as follows.

The structural diagram of the radio-photon airborne information line introducing nonlinear distortions into the radio signal is shown in Fig. 2.

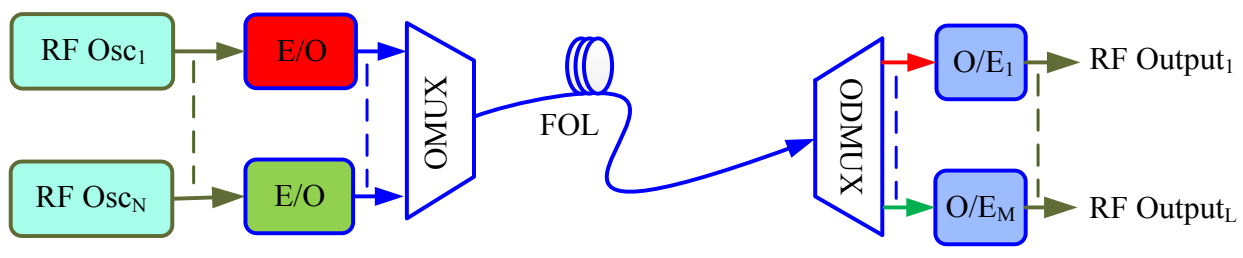

Fig. 2. Block diagram of a microwave optics frequency converter (RF Osc - radio frequency oscillator, E/O - electro-optical modulator, $\mathrm{O} / \mathrm{E}$ - optoelectronic receiver, OMUX - optical multiplexer, ODMUX- optical demultiplexer, FOL - fiber-optic communication line).

a) Laser source. To ensure the formation of single-mode optical radiation in the conditions of on-board satellite equipment, it is advisable to use a semiconductor compact and reliable laser with distributed feedback (Distributed Feedback Laser, DFB), the cavity of which consists of an active medium with an integrated periodic structure (Fig. 3).

Examples of structural diagrams of such a line are given in $[5,6]$. In [6], a laser DFB diode generates a single-mode optical radiation with a fixed wavelength near $\lambda=1550 \mathrm{~nm}$, a phase noise spectrum width near the center frequency of $10 \mathrm{MHz}$, and an optical power of up to $+10 \mathrm{dBm}$. In [5], it was noted that nonlinear intermodulation distortions of the optical envelope at the output of a third-order modulator can be caused by the asymmetry of the sideband transform in the Mach-Zehnder modulator (MZM) and the phase modulation of the polarized components in the intensity variation. In the same place, the authors pay attention to the manifestations of hysteresis in the characteristic of the MZM modulator, which lead to the appearance of additional intermodulation distortions of the transmitted signal. In [6], a two-band version of the MZM with carrier suppression was used, which forms a microwave envelope in the frequency band up to $22 \mathrm{GHz}$ with a reduced relative noise intensity $\mathrm{RIN} \approx-140 \mathrm{~dB} / \mathrm{Hz}$.

b) Chromatic dispersion in the fiber (dependence of the refractive index of the line material $n$ on the frequency of optical waves $f$ ). Its level is usually determined by the value 


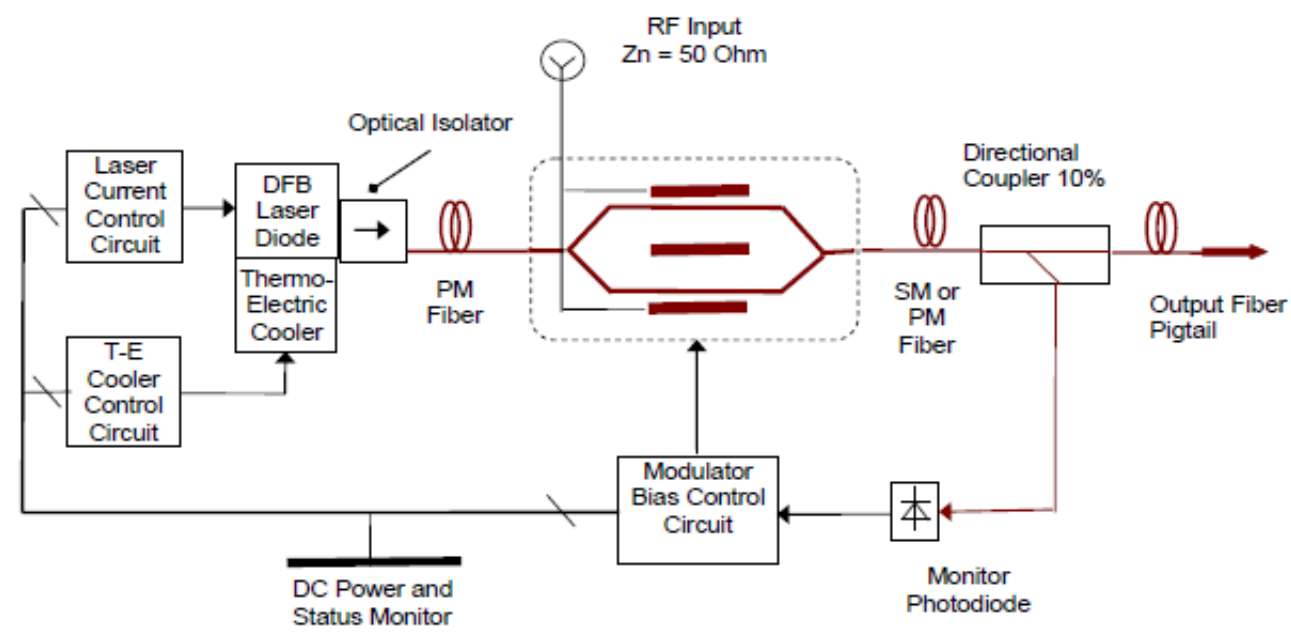

Fig. 3. Block diagram of the formation of an optical signal with analogue microwave modulation in intensity based on a DFB laser and an external Mach-Zehnder modulator for an onboard fiber optic link.

of the dispersion coefficient $\mathrm{D}_{\mathrm{xp}}$ at a wavelength of light in vacuum $\lambda=\mathrm{c} / f$, where $\mathrm{c} \approx 3 \cdot 10^{9} \mathrm{~m} / \mathrm{s}$ is the speed of light. Distinguish [4] its waveguide $\mathrm{D}_{\text {wave }}$ and material $\mathrm{D}_{\text {mat }}$ components $\mathrm{D}_{\mathrm{xp}}=\mathrm{D}_{\text {mat }}+\mathrm{D}_{\text {wave }}$ with the corresponding dispersion coefficients. Material caused by the dependence of the refractive index of optical glass on the wavelength, waveguide - is determined by changing the length of the wave propagation path inside the fiber, taking into account the profile of changes in the refractive index inside the fiber. The parameter $D_{\text {wave }}$ in the wavelength range $\lambda$ from 800 to $1600 \mathrm{~nm}$ is negative, and the value $\left.\mathrm{D}_{\text {mat }} \approx 122\left(1-\lambda_{\mathrm{ZD}} / \lambda\right)\left[\mathrm{ps} \cdot \mathrm{nm}^{-1} \cdot \mathrm{km}^{-1}\right)\right]$ changes sign depending on the ratio $\lambda / \lambda_{\mathrm{ZD}}$, where $\lambda_{\mathrm{ZD}}$ - wavelength of zero dispersion of the material. For pure silica glass, $\lambda_{\mathrm{ZD}}=1276 \mathrm{~nm}$, when doping is used, its value is shifted to a region near $\lambda=1550 \mathrm{~nm}(\mathrm{f}=193.55 \mathrm{THz})$, for which there are inexpensive laser sources and there is minimal broadening of the light pulse at the end of the fiber optic link. To reduce the effect of chromatic dispersion, a fiber with a predetermined wavelength offset of zero dispersion $\lambda_{\mathrm{ZD}}$ is used.

c) Polarization Mode Dispersion (PMD) [9]. In a single-mode fiber, it is caused by small deviations of the fiber core or its sheath from ideal central symmetry or the occurrence of mechanical stresses that lead to the propagation of orthogonally polarized components of light pulses with slightly different speeds. For long FOCLs, along with compensators for chromatic dispersion, technical solutions have also been developed to compensate for PMD or maintain its original polarization.

d) Intermode spread of group wave velocity values in a fiber [10]. This parameter is relevant only for multimode propagation mode. A single-mode fiber of the OS2 class (Low Water Peak, LWP), which, however, requires the use of a more complex and expensive laser source and modulator, is promising for use in on-board radio-photon lines at a high information flow rate.

e) Nonlinear signal distortion in the optical fiber. The level of these manifestations increases sharply with increasing radiation intensity (power per unit cross-sectional area) above a set threshold. They are associated with effects: stimulated light scattering on the acoustic phonons of the crystal lattice of the fiber (Brillouin (Mandelstam effect), Stimulated Brilouin Scattering - SBS); Raman scattering of light by optical phonons (Stimulated Raman Scattering - SRS); Kerr effect - the dependence of the refractive index of the medium on the electric field strength. As a result of the action of nonlinear distortions, additional noise and distortion may occur: the combination components of the 
four-wave (Four-Wave Mixing, FWM) mixing; self-phase modulation (SPM); spurious frequency modulation of pulses (chirping); cross-channel inter-channel modulation (suppression and blocking of adjacent channels); Modulation Instability (MI); the formation of a soliton (an optical pulse that propagates through the fiber without changing its shape with the equality between the dispersion of the phase velocity and phase self-modulation); jitter instability of the leading edge of the light pulse and its position relative to the boundaries of the clock interval.

According to [11], the attenuation of the light wave intensity as a result of the manifestation of the Brillouin effect for a wavelength of $\lambda=1550 \mathrm{~nm}$ at a line length of $\mathrm{L}=22 \mathrm{~km}$ is about $-40 \mathrm{~dB}$ with a threshold power of one-unit compression of $3 \mathrm{dBm}$. Therefore, for the length of the fiber optic link not more than a few tens of meters, characteristic of onboard space equipment, the SBS effect can be neglected. The attenuation of the intensity due to Raman Raman scattering SRS does not exceed $-60 \mathrm{~dB}$ for a threshold power of $30 \mathrm{dBm}$ for the same parameters of the line and wavelengths of light. Calculations and measurement results for other nonlinear effects in the optical fiber (phase selfmodulation, modulation instability) show that the relative level of transient and Raman interference in airborne FOCLs does not exceed $-70 \mathrm{~dB}$.

The FWM four-wave mixing effect in optical fibers generates spectral sidebands that are distant from the pump frequency up to $100 \mathrm{THz}$. It occurs in a fiber optic link with a multi-frequency signal with frequencies $f_{i}, f_{j}, f_{k}$ due to the appearance of combinational components with frequencies $f_{i}+f_{j}-f_{k}$, which interfere with side channels. Such a parameter is convenient for taking into account cross-channel interchannel distortions during propagation along a fiber-optic signal with WDM wavelength division multiplexing or with O-OFDM optical orthogonal frequency multiplexing [4,7,10]. The level of interference from the FWM effect is not significantly related to the line length and may turn out to be comparable with that delivered by other mechanisms in the case of phase matching of pump sources for fiber optic links with multiple inter-channel frequency spacing, when the Raman frequencies of even second order fall on one of the active channels. Thus, the FWM four-wave mixing mechanism is not noticeable for airborne FOCLs with a small number of channels.

Frequency and nonlinear distortions in the photodetector are considered in detail in [11]. It was found there that in order to reduce their relative influence, it is advisable to provide a high signal-to-noise ratio at its input. In particular, it was shown that the frequency dependence of the frequency transfer coefficient in the p-i-n-photodetector has a pulsating character with a span of several decibels, and its effective passband decreases markedly with increasing level of incident optical power. This is caused by inertial processes in the semiconductor structure of the photodiode. To increase the sensitivity of the photodetector, reduce non-linear manifestations, and expand the bandwidth of the frequencies transmitted by the photodetector, it is necessary to use broadband matching of the differential resistance of the photodiode with the transmission line, which is done using transimpedance cascades.

[3] Distributed Feedback laser (DFB) lasers [3] appear to be the most promising [3] as the sources of the carrier vibrations of the optical range for onboard radio-photon communication lines. To enter information, a method of analog modulation in intensity is recommended using an external electro-optical modulator MZM based on the MachZehnder interferometer (Modulator Mach-Zehnder) [7]. Measures were developed and presented in [11] to minimize the interfering manifestations of dispersion and nonlinear distortions of transmitted signals in backbone FOCLs using predistorting cascades. 


\section{Conclusion}

When constructing on-board satellite equipment, it is advisable to use RF FOCLs and optical range components [12], given the extensive experience in linearizing the intermodulation amplitude and energy characteristics of signal transmission paths accumulated during the creation of microwave power amplifiers

\section{References}

1. A. A. Emelyanov, M. E. Belkin, N.V. Toporkov, V. A. Masnoy, Features of the construction of an onboard fiber-optic synchro network, Radio Engineering, №8, pp. $121-125$ (2017)

2. C. Ciminelli, C. M. Armenise, F. Dell'Olio, Photonics in space: advanced photonic devices and systems, (World Scientific, New Jersey, 2016)

3. A. D. Shcherbinin, L. A. Belov, Microwave Optics Technologies in Satellite Repeaters, 2019 Systems of Signal Synchronization, Generating and Processing in Telecommunications (SYNCHROINFO). - Yaroslavl, Russia. DOI: 10.1109/ SYNCHROINFO.2019.8813963 (1-3 July 2019)

4. E. L. Portnov, A. L. Senyavsky and B. P. Khromoi, Metrology in optical telecommunication systems (Goryachaya linia - Telekom, Moscow, 2019)

5. S. I. Ivanov, A. P. Lavrov, I. I. Saenko, Study of the characteristics of transmitter modules with internal and external modulation in analog fiber-optic transmission lines of microwave radio signals, Microwave Electronics and Microelectronics. v. 2. pp. 69 -73 (2016)

6. V. M. Afanasyev, R. S. Ponomarev Афанасьев, В. М. Electro-optical amplitudeoptical Mach-Zehnder modulators based on lithium niobate, their modifications and modulation formats //B. М. Афанасьев, Р. С. Пономарев, Applied Photonics, № 5, v. 4, pp $337-360$ (2017)

7. Urik W. JM, McKinney D.D., Williams K.D., Basics of microwave photonics (Technosphera,Moscow, 2016)

8. A. D. Shcherbinin, L. A. Belov, Radio photon technologies in on-board satellite, Systems for synchronizing, generating and processing signals in infocommunications (to be published)

9. E. L. Portnov, Polarization mode dispersion on a fiber optic transmission line , T-Comm, №9, pp. 62 - 64 (2014)

10. V.N. Listvyn, D.V. Shvyrkov, Optical fibers for communication lines (LESARart, Moscow, 2003)

11. V. G. Fokin, R.Z. Ibrahimov, Optical systems with terabit and petabit transmission rates (Goryachaya linia - Telekom, Moscow, 2017)

12. P. S. Devgan, Applications of Modern RF Photonics (Artech House, London, 2019) 